\title{
Theory and Phenomenology of Vector Mesons in Medium
}

\author{
Ralf Rapp\$ \\ Cyclotron Institute and Physics Department, Texas A\&M University, College \\ Station, Texas 77843-3366, U.S.A.
}

\begin{abstract}
Electromagnetic probes promise to be direct messengers of (spectral properties of hot and dense matter formed in heavy-ion collisions, even at soft momentum transfers essential for characterizing possible phase transitions. We examine how far we have progressed toward this goal by highlighting recent developments, and trying to establish connections between lattice QCD, effective hadronic models and phenomenology of dilepton production.
\end{abstract}

\section{Introduction}

High-energy heavy-ion physics aims at investigating the properties of strongly interacting matter under extreme conditions, at much higher temperatures $(T)$ and baryon densities $\left(\varrho_{B}\right)$ than accessible in atomic nuclei. A major goal is to establish the existence of new states of matter characterized by the restoration of the spontaneously broken chiral symmetry and the deconfinement of color charges, as predicted by Quantum Chromodynamics (QCD). Hadronic observables are widely (and successfully) used to infer the composition and collective expansion of the highly excited system and thus determine its bulk properties, i.e., equation of state (EoS). Direct information on the degrees of freedom and spectral properties of the matter are harder to obtain, especially at soft scales typical for the phase transition, $\sim T_{c}, \Lambda_{\mathrm{QCD}}$, up to the chiral scale $\Lambda_{\chi} \sim 4 \pi f_{\pi}$ $\left(f_{\pi}=92 \mathrm{MeV}\right.$ : pion decay constant). Electromagnetic (e.m.) probes (real and virtual photons) provide a unique opportunity to study the $J^{P}=1^{-}$response of the strongly interacting medium [1, 2, 3, 4], defined by the pertinent retarded correlation function,

$$
\Pi_{\mathrm{em}}^{\mu \nu}\left(q ; \mu_{B}, T\right)=-i \int d^{4} x \Theta(t) \mathrm{e}^{i q x}\left\langle\Omega\left|j_{\mathrm{em}}^{\mu}(x) j_{\mathrm{em}}^{\nu}(0)\right| \Omega\right\rangle
$$

$\left(|\Omega\rangle\right.$ : finite $T, \mu_{B}$ ground state). From $e^{+} e^{-} \rightarrow$ hadrons one knows that, in the vacuum, the low-mass $\left(M \lesssim \Lambda_{\chi}\right)$ e.m. spectral function is saturated by light vector mesons,

$$
\operatorname{Im} \Pi_{\mathrm{em}} \sim\left[\operatorname{Im} D_{\rho}+\frac{1}{10} \operatorname{Im} D_{\omega}+\frac{1}{5} \operatorname{Im} D_{\phi}\right],
$$

which also illustrates the dominant role of the $\rho$. In vector-dominance models, medium modifications are thus encoded in the vector-meson propagators, $D_{V}(V=\rho, \omega, \phi)$.

$\S$ email: rapp@comp.tamu.edu 
The study of vector mesons in hadronic matter should be placed in a broader context. This includes questions of the type: (1) Do $\rho, \omega$ and $\phi$ mesons behave alike? (2) Are hadronic interactions sufficient to account for medium effects or are "intrinsic" $T$ - and $\mu_{B}$-dependencies of the parameters of the effective Lagrangian to be included [5, 6]? (3) How do vector-meson spectral functions change across the phase diagram (i.e., baryon- vs. meson-induced modifications)? (4) What is the impact of medium-modified hadron properties on the EoS and its chemical composition? (5) What are the connections between vector spectral functions and (chiral) order parameters (quark and gluon condensates, $f_{\pi}$, susceptibilities, ...), and how can they be exploited? The nonperturbative nature of these questions requires the use of effective (chiral) models with careful constraints from both first principles (symmetries, lattice QCD) and experiment. Phenomenological applications to electromagnetic observables in heavy-ion collisions can then pave the way to deduce the onset and realization of chiral symmetry restoration, a key prediction of QCD.

In this paper, we discuss 3 basic elements (lattice QCD, effective models and phenomenology) to address the above issues. Sec. 2 recalls relations between e.m. spectral functions and chiral order parameters via susceptibilities and sum rules. Sec. 3 gives a brief survey on vector mesons in hot/dense hadronic matter. Sec. 4 assesses ingredients for studying dilepton spectra in heavy-ion collisions, with applications to,

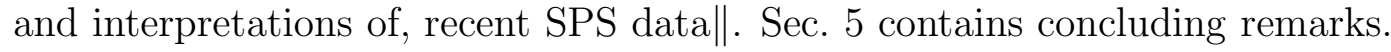

\section{Vector Correlators from Lattice QCD and Sum Rules}

The basic quantity which, in principle, contains all the information about an interacting system at finite $T$ and $\mu_{B}$ (baryon chemical potential) is the thermodynamic potential,

$$
\Omega\left(\mu_{B}, T\right)=-T \log \mathcal{Z}=-P V
$$

defined in terms of the partition function, $\mathcal{Z}\left(P\right.$ : pressure). Its $T$ and $\mu_{B}$ derivatives give rise to the EoS (entropy, energy-/density), while hadronic correlation functions follow from expectation values of suitable currents, cf. Eq. (11). The EoS is routinely computed in lQCD, but the formulation of IQCD in Euclidean (imaginary) time renders it more difficult to extract spectral functions in the timelike regime. A valuable interface of bulk and microscopic quantities is provided by susceptibilities, which are 2. order derivatives of $\Omega$ that can be related to the spacelike (screening) limit of pertinent correlators,

$$
\chi_{\alpha} \sim \frac{\partial^{2} \Omega}{\partial \mu_{\alpha}^{2}} \sim \Pi_{\alpha}\left(q_{0}=0, q \rightarrow 0\right) .
$$

Fig. 1 shows a recent lQCD computation of the $T$-dependence of light quark-number susceptibilities in the isoscalar $(\alpha=q)$ and isovector $(\alpha=I)$ channel, carrying the quantum number of the $\omega$ and $\rho$ meson, respectively. With increasing $\mu_{q}$ (evaluated via a Taylor expansion), the isovector channel behaves smoothly while the isoscalar one develops a

|| Medium effects on vector mesons in elementary (photo-/electro-/hadro-) production off nuclei have been discussed in excellent review talks by Djalali [7] and Metag [8] and will not be reiterated here. 

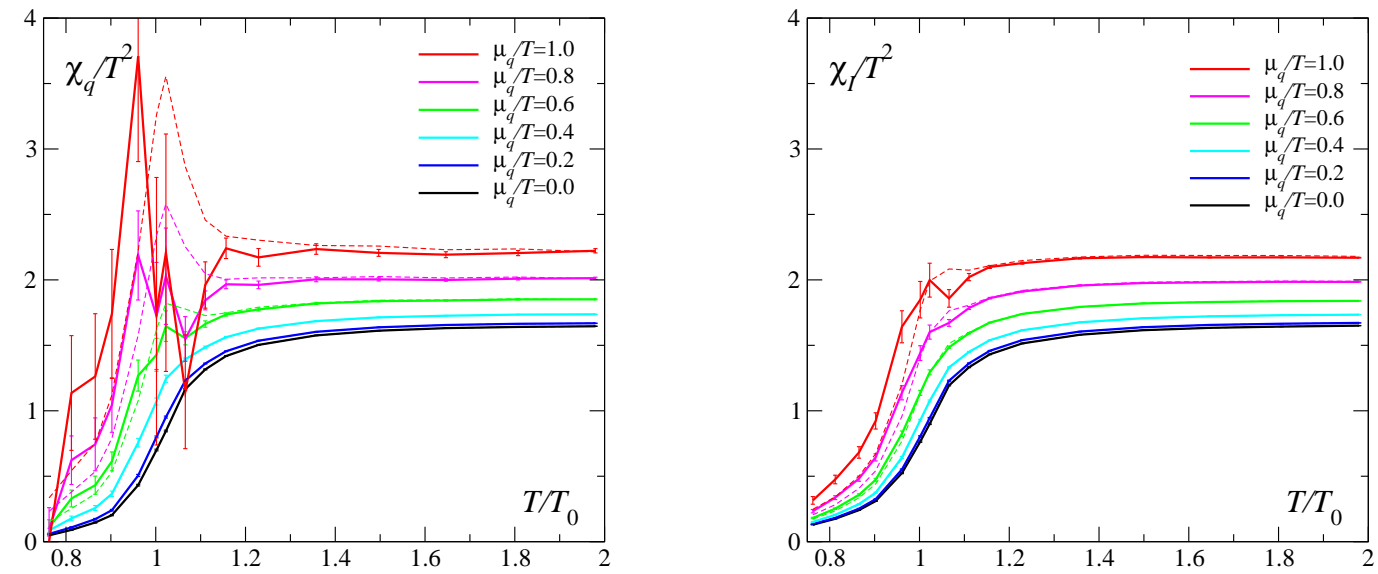

Figure 1. Isoscalar (left) and isovector (right) quark-number susceptibility for various quark chemical potentials, $\mu_{q}=\mu_{B} / 3$, as computed in unquenched lattice QCD 9 .

pronounced peak. This could be related to the approach to a critical endpoint in the phase diagram, possibly induced by the effect of a $\sigma-\omega$ mixing.

A powerful tool to relate chiral order parameters to (in-medium) $V$-meson spectral functions are sum rules (SRs). In QCD-SRs a (subtracted) dispersion relation for a correlation function is formulated for spacelike momenta $q^{2}=-Q^{2}<0$ [10],

$$
\Pi_{\alpha}\left(Q^{2}\right)=\Pi_{\alpha}(0)+c Q^{2}+Q^{4} \int \frac{d s}{s^{2}} \frac{\operatorname{Im} \Pi_{\alpha}(\mathrm{s})}{s+Q^{2}} .
$$

The spectral function on the right-hand-side is related to quark and gluon condensates which arise as nonperturbative coefficients within an operator-product expansion (OPE) in $1 / Q^{2}$ of $\Pi_{\alpha}\left(Q^{2}\right)$ on the left-hand-side. The condensates on the OPE side generally decrease in the medium, which, in turn, requires a softening of the spectral function under the dispersion integral. The softening can be satisfied by both broadening and a downward mass shift [11, 12]. For $\omega$ and $\rho$ mesons the OPE side is very similar (governed by 4-quark condensates), while the subtraction constant differs considerably, $\Pi_{\rho}(0)=\Pi_{\omega}(0) / 9$, implying stronger medium effects (softening) on the $\rho$ than on the $\omega$.

For the vector-isovector $(\rho)$ channel (which dominates the dilepton rate, cf. eq. (2)), so-called Weinberg and DMO sum rules [13, 14] are of special interest. Using current algebra, one can relate moments of the difference between vector and axialvector spectral functions to chiral order parameters. In the chiral limit $\left(m_{\pi}=0\right)$ one has

$$
\begin{aligned}
& f_{n}=-\int_{0}^{\infty} \frac{d s}{\pi} s^{n}\left[\operatorname{Im}_{V}(s)-\operatorname{Im}_{A}(s)\right], \\
& f_{-2}=f_{\pi}^{2} \frac{\left\langle r_{\pi}^{2}\right\rangle}{3}-F_{A}, \quad f_{-1}=f_{\pi}^{2}, \quad f_{0}=0, \quad f_{1}=-2 \pi \alpha_{s}\langle\mathcal{O}\rangle
\end{aligned}
$$

$\left(r_{\pi}\right.$ : pion charge radius, $F_{A}$ : radiative pion decay constant, $\langle\mathcal{O}\rangle$ : 4-quark condensate). Weinberg sum rules remain valid at finite $T$ [15], demonstrating that chiral restoration requires degeneracy of the entire spectral functions. Combining lQCD computations of order parameters with effective model calculations of spectral functions thus provides a very promising synergy for deducing chiral restoration from experiment [16]. 

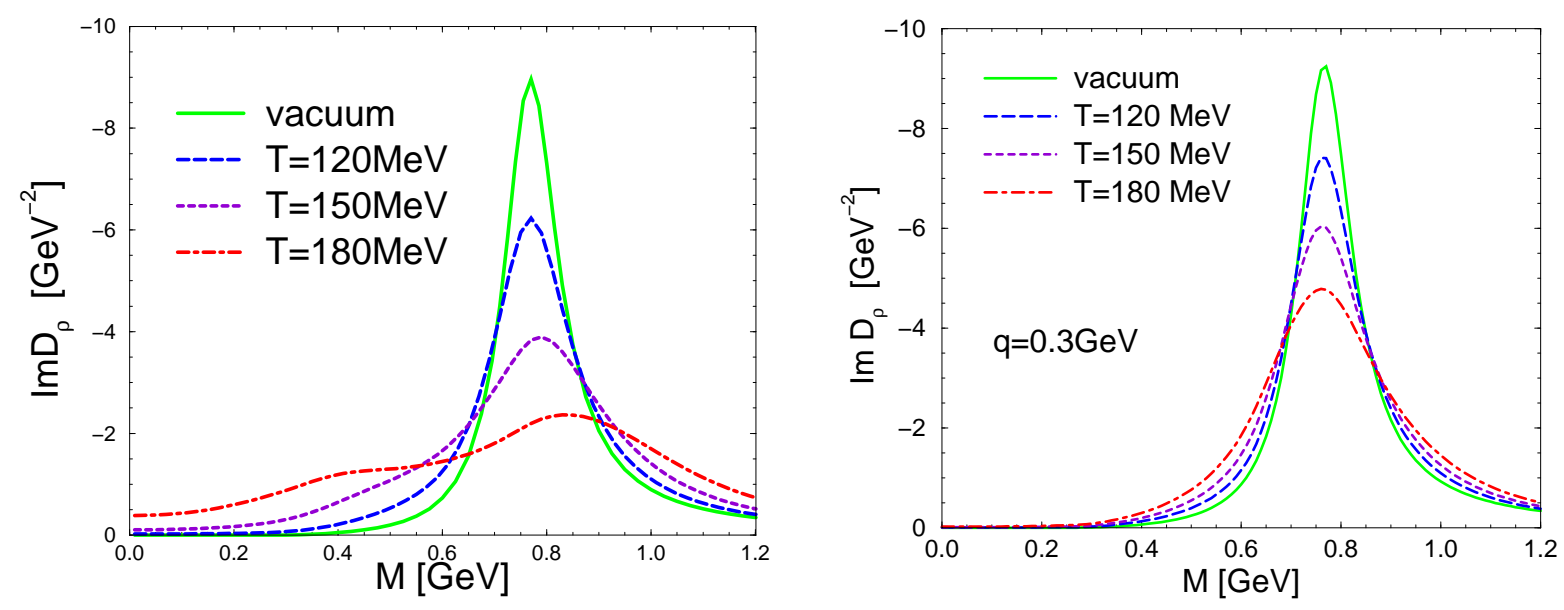

Figure 2. $\rho$-meson spectral function in a hadronic many-body approach. Left panel: hot hadronic matter [17] at fixed $\mu_{B}=330 \mathrm{MeV}$ (approximately reflecting CERNSPS conditions $)$ corresponding to $\left(\varrho_{B} / \varrho_{0}, T / \mathrm{MeV}\right) \simeq(0.1,120),(0.7,150),(2.6,180)$; right panel: hot meson gas (no anti-/baryons) [18].

\section{Hadronic Spectral Functions}

A commonly used approach to evaluate in-medium $V$-meson spectral functions is hadronic many-body theory [1]. The key quantity figuring into the $V$-meson propagator,

$$
D_{V}\left(M, q ; \mu_{B}, T\right)=\left[M^{2}-m_{V}^{2}-\Sigma_{V P}-\Sigma_{V B}-\Sigma_{V M}\right]^{-1},
$$

is the selfenergy, $\Sigma_{V}$, which can be calculated from effective interactions integrated over the momentum distributions of mesons $(M=\pi, K, \rho, \ldots)$ and baryons $(B=N, \Lambda, \Delta, \ldots)$ in the heat bath $\left(\Sigma_{V P}\right.$ encodes medium-modified $\pi \pi, 3 \pi, K \bar{K}$ decays). The interaction vertices ought to be compatible with gauge and chiral invariance and constrained by resonance decay widths and scattering data in the vacuum. Typical results for the $\rho$ spectral function are shown in Fig. 2, Prominent features are a strong broadening with little mass shift and the prevalence of anti-/baryonic effects, especially below the free $\rho$ mass. When extrapolated to the expected phase boundary, the $\rho$ spectral function "melts" and resembles the form and magnitude of the perturbative $q \bar{q}$ correlator. In cold nuclear matter, it is compatible with QCD sum rule analysis [11] and qualitatively consistent with the new CLAS data for photoproduction off nuclei at JLab 7 .

In Ref. [19, the $\Sigma_{V}$ 's have been evaluated in terms of empirical scattering amplitudes as constructed from resonance dominance at low energies and Regge-type behavior at high energies, while the real parts have been calculated using dispersion relations. It is satisfying to see that the resulting $\rho$ spectral function agrees quite well with the many-body approach, cf. left panel of Fig. 3] (the largest discrepancy emerges toward the $2 \pi$ threshold; also, some care has to be taken in identifying nucleon and baryon densities at finite $T$ ). The $\omega$ spectral function (right panel of Fig. 3) also broadens considerably (with little mass shift), but less than the $\rho$, which is in line with 

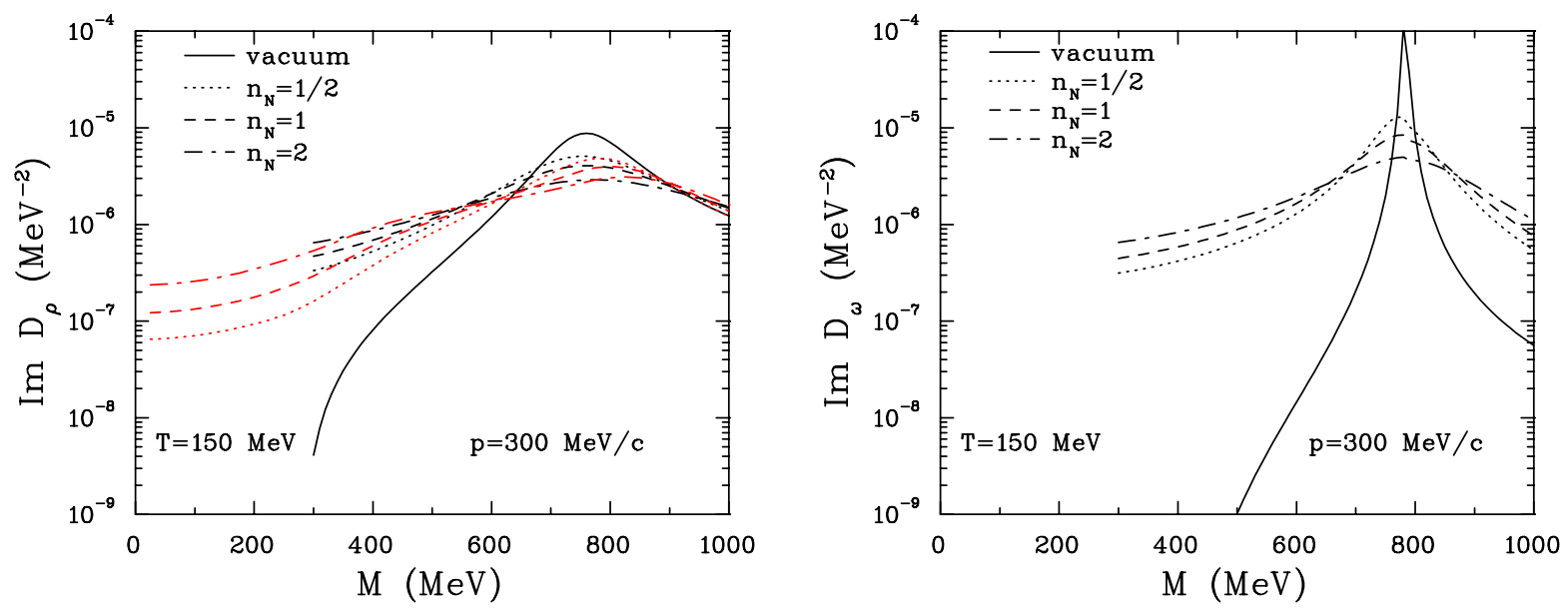

Figure 3. $V$-meson spectral functions in a hot $\pi-N$ gas based on empirical scattering amplitudes constrained by resonances and Regge behavior [19]. Left panel: $\rho$ meson, compared to hadronic many-body calculations of Fig. 2. Right panel: $\omega$ meson.

the expectation from QCD sum rules (recall the discussion after eq. (5)).

Within the Hidden Local Symmetry approach, it has been suggested to identify the chiral partner of the (longitudinal) $\rho$ with the $\pi$ (rather than $a_{1}$ ) [5]. When applied within a finite- $T$ loop expansion, and after matching the hadronic axial-/vector correlators to pQCD in the spacelike regime, one finds a dropping $\rho$ mass as well as a violation of vector dominance. The finite- $T$ e.m. formfactor, which enters into the dilepton production rate, clearly exhibits the downward moving $\rho$ peak [20]. It would be interesting to see whether this feature persists when including the effects of baryons.

\section{Dilepton Phenomenology in Heavy-Ion Collisions}

The dilepton emission rate is directly proportional to the e.m. spectral function [21],

$$
\frac{d N_{l l}}{d^{4} x d^{4} q}=-\frac{\alpha_{\mathrm{em}}^{2}}{\pi^{3} M^{2}} f^{B}\left(q_{0} ; T\right) \operatorname{Im}_{\mathrm{em}}\left(M, q ; \mu_{B}, T\right) .
$$

Assuming the medium in a heavy-ion collision to kinetically equilibrate, thermal dilepton spectra can be calculated by convolution over a realistic space-time model. Obviously, the latter must be consistent with hadronic chemistry (evolution of $\mu_{B}$ and $T$ ) and $p_{T}$ spectra (collective expansion). However, depending on centrality and invariant mass or $q_{T}$ of the lepton pair, nonthermal effects/sources, such as Drell-Yan annihilation and final-state decays (including correlated open-charm), may not be neglected.

In the upper panels of Fig. 4 thermal fireball calculations employing $V$-meson spectral functions from hadronic many-body theory [22] are compared to recent NA60 data (where improved experimental accuracy enabled a subtraction of final-state hadron decay contributions, except for $\rho$ and charm decays) [23]. The low-mass region is well described by the in-medium spectral functions (upper left panel), supporting the predicted $\rho$ line shape (QGP emission is rather small). When switching off baryonic effects (or all medium effects), the spectra are incompatible with the data (upper right 

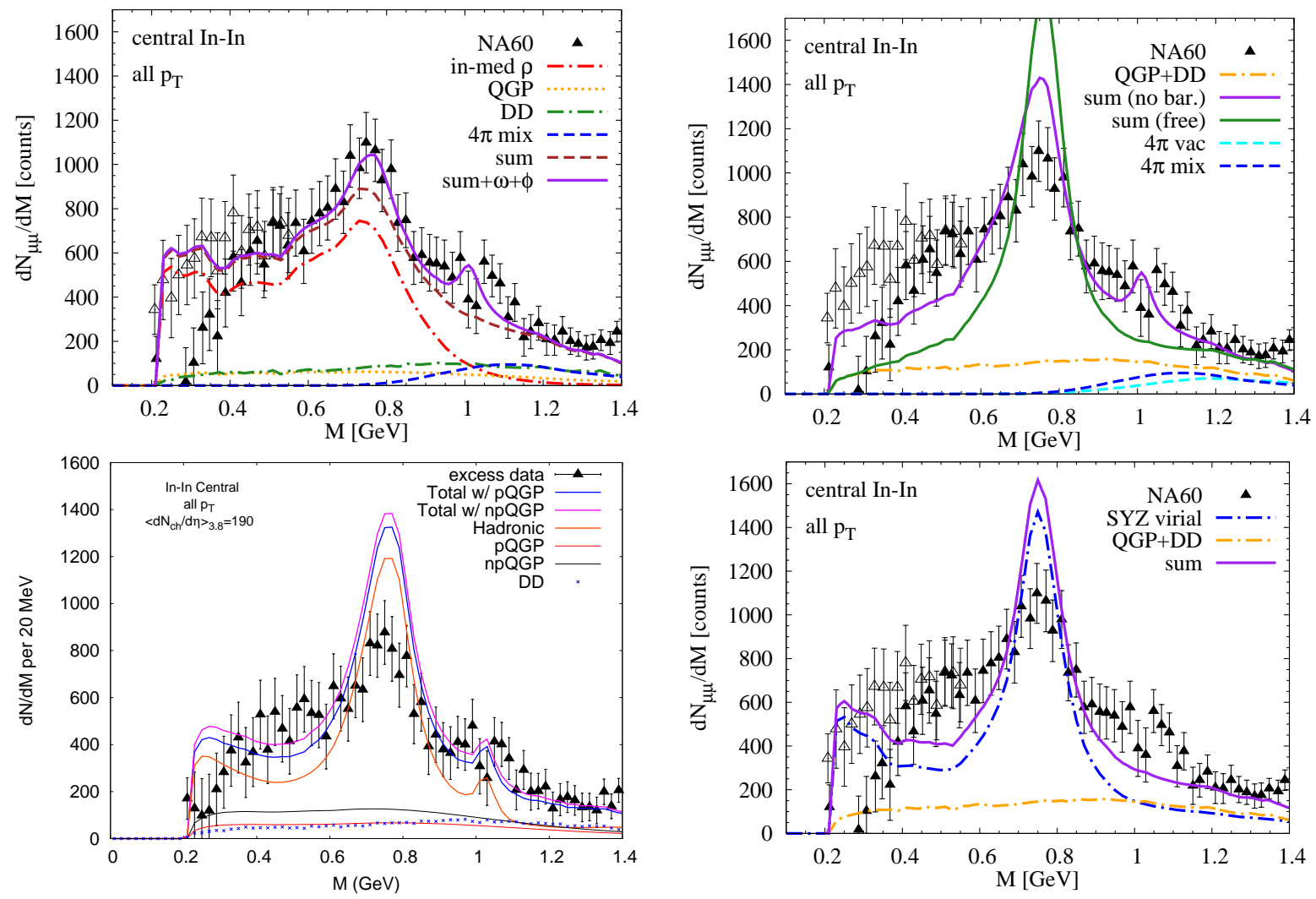

Figure 4. NA60 dimuon invariant-mass spectra in central 158 AGeV In-In collisions [23, compared to thermal fireball emission based on hadronic many-body theory and $q \bar{q}$ annihilation in a QGP $[22$ (upper panel; left: full results, right: without baryon effects and when using the free e.m. spectral function). Lower panels: thermal spectra using e.m. rates from a chiral virial expansion within a hydrodynamic evolution [26] (left) and a thermal fireball 22] (right).

panel). In fact, the space-time integrated dilepton line shapes for different scenarios reflect the changes of the $\rho$ spectral function in Fig. 2, confirming good sensitivity to medium effects. At masses above $1 \mathrm{GeV}$, the free e.m. correlator mostly couples to 4-pion states. Inclusion of this contribution in the fireball emission leads to reasonable agreement with the observed enhancement at $M \geq 1 \mathrm{GeV}$ (effects of leading- $T$ vectoraxialvector mixing [24] increases the $4 \pi$ yield slightly). Note that the relative strength of the various thermal sources is fixed; the absolute yield is sensitive to the fireball lifetime, which in Fig. 4 amounts to about $7 \mathrm{fm} / \mathrm{c}$ (the initial/final temperatures are $\left.T_{\mathrm{i}, \mathrm{fo}} \simeq 195,120 \mathrm{MeV}\right)$. The lower panels of Fig. 4 display results of using the in-medium e.m. spectral function calculated in a 3-flavor chiral reduction formalism [25], which couples chiral Ward identities with a low-density expansion using empirical $\rho-/ \gamma-N$ and $\rho$ - $\pi$ scattering amplitudes. The low- and intermediate-mass enhancement is well described, but the lack of $\rho$-broadening (inherent to the low-density expansion) leads to an overestimate around the free $\rho$ mass. The spectra obtained from the hydrodynamic evolution used in Ref. [26] (left panel) agree well with the fireball approach of Ref. [22] (right panel). This also holds for the (smallness of the) perturbative QGP contribution. 

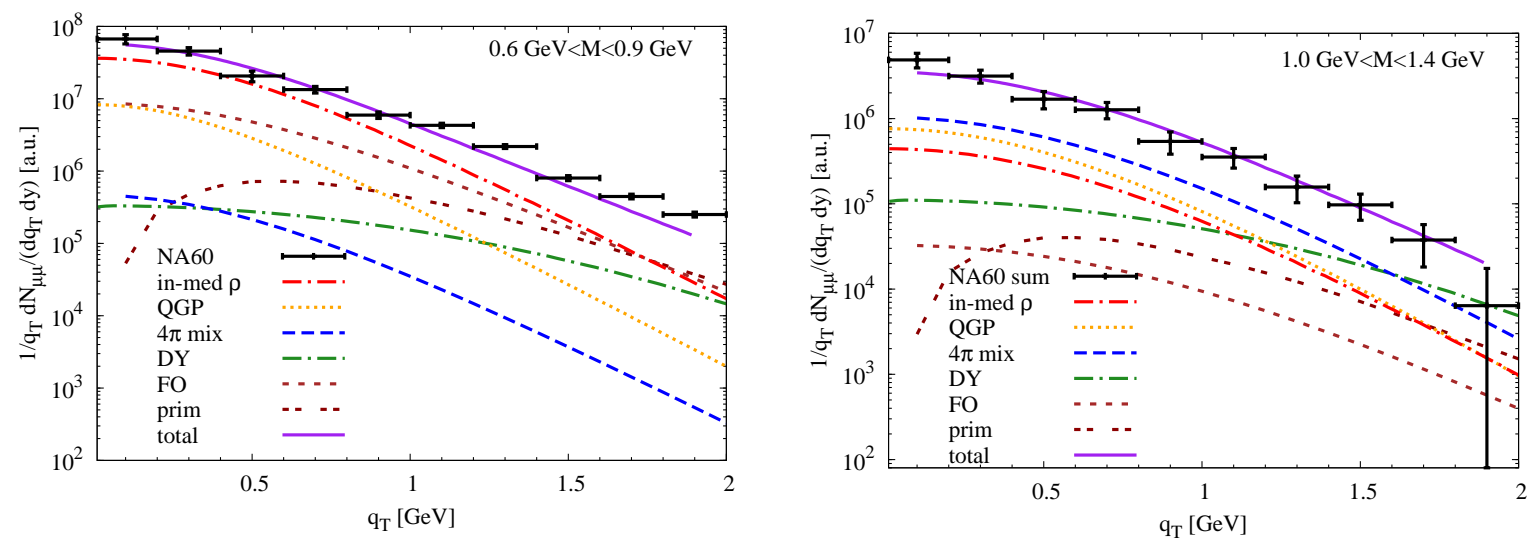

Figure 5. Dimuon $q_{T}$ spectra in semicentral $158 \mathrm{AGeV}$ In-In collisions 28 for mass bins $0.6 \mathrm{GeV}<M<0.9 \mathrm{GeV}$ (left) and $1.0 \mathrm{GeV}<M<1.4 \mathrm{GeV}$ (right), compared to thermal fireball calculations with in-medium $\rho, \omega, \phi$ spectral functions, $4 \pi$ annihilation, QGP emission, thermal-freezeout and primordial $\rho$ 's, as well as DrellYan annihilation 31.

On the contrary, in Ref. [27] QGP radiation is the dominant source above $M \simeq 0.9 \mathrm{GeV}$.

Additional information on the nature of the excess radiation in heavy-ion collisions can be obtained from pair- $q_{T}$ spectra, especially when differential in mass, see Fig. 5. At low $q_{T}$ the spectra are dominated by thermal radiation (either $2 \pi$ or $4 \pi$ annihilation), but the composition becomes more involved at higher $q_{T}$. In the $\rho$-mass region, $\rho$ decays at freezeout (subject to a large flow-induced blueshift, with in-medium spectral shape) as well as primordial $\rho$ 's from minijets escaping the medium (including a Cronin effect, with vacuum spectral shape) become significant. Note that the emission kinematics of these sources actually differs from thermal radiation by a time dilation factor $\gamma=q_{0} / M$. In the $\rho$-mass bin, the theoretical $q_{T}$ spectra appear somewhat too soft (as more clearly revealed in a local slope analysis [28]), cf. also Ref. [27. An interesting possibility is that viscosity effects in the later hadronic stage render hadron spectra harder [29]. Transverse-momentum and centrality dependencies certainly deserve further study.

The CERES/NA45 collaboration has recently published dielectron excess spectra for central $\mathrm{Pb}(158 \mathrm{AGeV})+\mathrm{Au}$ [30]. Albeit inferior in statistics to the NA60 dimuons, the small electron mass allows access to the mass region below the $2 \pi$ threshold, where theory predicts a large enhancement (the data might show first indications of this). In addition, in larger collision systems, the relative size of nonthermal contributions is suppressed. Hadronic many-body calculations describe the CERES spectra well.

\section{Conclusions}

E.m. probes are progressively improving our understanding of microscopic properties of hot and dense matter. In this paper we have focused on soft emission, which predominantly relates to the physics of in-medium vector mesons. Hadronic models, when carefully constrained, predict a strong in-medium broadening ("melting") of the 
$\rho$ resonance which has withstood a substantial increase in accuracy in recent dilepton measurements at the SPS. This also corroborates the notion of (anti-) baryons as the major agents of medium effects, which is expected to hold even at RHIC and LHC, raising intriguing questions on the nature of chiral restoration. The determination of the in-medium axialvector spectral function remains a pressing issue. It can and will be addressed by constructing realistic chiral models in a hot and dense medium, which will serve as a bridge between order parameters (as computed, e.g., in lattice QCD) and experimental data, by utilizing chiral and QCD sum rules.

\section{Acknowledgment}

I thank the conference organizers for the invitation to an exciting meeting, and $\mathrm{H}$. van Hees for fruitful collaboration. This work was supported in part by a U.S. National Science Foundation CAREER award under grant no. PHY-0449489.

\section{References}

[1] Rapp R and Wambach J 2000 Adv. Nucl. Phys. 251

[2] Alam J, Sarkar S, Roy P, Hatsuda T and Sinha B 2001 Ann. Phys., NY 286159

[3] Cassing W and Bratkovskaya E L 1999 Phys. Rep. 30865

[4] Gale C 2006 Nucl. Phys. A $\mathbf{7 7 4} 335$

[5] Harada M and Yamawaki K 2003 Phys. Rep. 3811

[6] Brown G E and Rho M 2004 Phys. Rep. 398301

[7] Djalali C 2007 these proceedings

[8] Metag V 2007 these proceedings

[9] Allton C R et al 2005 Phys. Rev. D 71054508

[10] Shifman M A, Vainshtein A I and Zakharov V I 1979 Nucl. Phys. B 147385

[11] Leupold S, Peters W and Mosel U 1998 Nucl. Phys. A 628311

[12] Ruppert J, Renk T and Müller B 2006 Phys. Rev. C 73034907

[13] Weinberg S 1967 Phys. Rev. Lett. 18507

[14] Das T, Mathur V S and Okubo S 1967 Phys. Rev. Lett. 19859

[15] Kapusta J I and Shuryak E V 1994 Phys. Rev. D 494694

[16] David G, Rapp R and Xu Z 2006 arXiv:nucl-ex/0611009

[17] Rapp R and Wambach J 1999 Eur. Phys. J. A 6415

[18] Rapp R and Gale C 1999 Phys. Rev. C 60024903

[19] Eletsky V L, Belkacem M, Ellis P J and Kapusta J I 2001 Phys. Rev. C 64035202

[20] Harada M and Sasaki C 2006 arXiv:hep-ph/0608237.

[21] McLerran L D and Toimela R 1985 Phys. Rev. C 31545

[22] van Hees H and Rapp R 2006 Phys. Rev. Lett. 97102301

[23] NA60 Collaboration [Arnaldi R et al ] 2006 Phys. Rev. Lett. 96162302

[24] Dey M, Eletsky V L and Ioffe B L 1990 Phys. Lett. B 252620

[25] Steele J V, Yamagishi H and Zahed I 1997 Phys. Rev. D 565605

[26] Dusling K, Teaney D and Zahed I 2006 arXiv:nucl-th/0604071

[27] Renk T and Ruppert J 2006 arXiv:hep-ph/0612113.

[28] Damjanovic S [NA60 Collaboration] 2007 arXiv:nucl-ex/0701015

[29] Teaney D 2007 these proceedings.

[30] CERES Collaboration [Adamova D et al ] 2006 arXiv:nucl-ex/0611022

[31] van Hees H and Rapp R 2007 arXiv:hep-ph/0701167 